\title{
Experiencias del diagnóstico comunitario participativo en Dzityá, Yucatán, México
}

\author{
Didier Francisco Aké Canul ${ }^{1, a, f}$, Josefina Inés Maldonado Borges ${ }^{2, b, f}$, Rocio Laviada Delgadillo, \\ Maricela Balam Gómez ${ }^{4, \mathrm{~d}, \mathrm{f}}$, Rosa María Yáñez González ${ }^{5, \mathrm{e,f}}$, Evelyn María Díaz Marfil ${ }^{6, \mathrm{~d}, \mathrm{f}}$
}

\section{RESUMEN}

Objetivos: identificar los determinantes que afectan las condiciones de salud de una población desde la perspectiva de los actores sociales. Abordaje Metodológico: se trata de un diagnóstico comunitario participativo, realizado en la localidad de Dzityá, perteneciente al municipio de Mérida, Yucatán, México, durante los meses de enero a abril de 2014, de tipo exploratorio, basado en la comunidad y con enfoque metodológico mixto. Se privilegió el enfoque cualitativo para obtener información a través del análisis del discurso de 13 entrevistas semiestructuradas, y 2 ejercicios de cartografía sociala actores clave de la localidad. Resultados: la contaminación ambiental generada por las partículas de polvo de piedra, originadas de la actividad artesanal, fue el determinante que la comunidad eligió como prioritario de atención, por los efectos que esta provoca en la salud de los pobladores. Conclusiones: con base a la perspectiva de los actores sociales, en esta comunidad se encontraron diferentes comorbilidades y factores de riesgo de tipo ambiental y social, que pueden ser modificables a través de intervenciones eficientes y oportunas con enfoque de promoción a la salud; mediante la voluntad política y la acción conjunta del sector salud, académico y la comunidad.

PALABRAS CLAVE: Diagnóstico de la situación de salud, participación comunitaria, investigación participativa basada en la comunidad, Yucatán, México.

\section{Experiences of the community participative diagnosis in Dzitya, Yu- catan, Mexico}

\section{SUMMARY}

Objectives: identify determinants that affect the health conditions of a population from the perspective of social actors. Material and methods: this is a participative community diagnosis, conducted in the town of Dzityá, in the municipality of Merida, Yucatan, Mexico, during the months of January to April 2014, it was exploratory, cross-sectional and community based. The qualitative approach was privileged to obtain information through discourse analysis of 13 semi-structured interviews, and 2 social mapping exercises with key actors of the town. Results: environmental pollution from stone dust particles originated from the artisanal activity, was the determining factor that the community chose as priority attention due to the effects that this has on the health of residents. Conclusions: with base to the perspective of the social actors, in this community were found various comorbidities and environmental and social risk factors, which can be modified through efficient and timely interventions with a focus on health promotion; as well as through political will and joint action of health, academic and active participation of the community.

KEYWORDS: Diagnosis of health situation, consumer participation, primary health care, community-based participatory research, Yucatán.

Facultad de Enfermería de la Universidad Autónoma de Yucatán. Yucatán, México

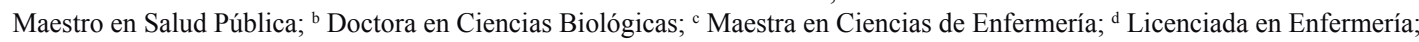

Doctora en Educación; ${ }^{\mathrm{f}}$ Docente investigador. 


\section{INTRODUCCIÓN}

La salud es un fenómeno dinámico afectado por múltiples cambios y circunstancias a los que se enfrentan las poblaciones los cambios pueden ser económicos, demográficos, ambientales, políticos, epidemiológicos, entre otros (1). Para conocer los factores que afectan a una población, la salud pública se vale del Diagnóstico de Salud Poblacional, el cual considera los daños a la salud, los determinantes de la salud y la respuesta social organizada (2).

El Diagnóstico de Salud Poblacional pertenece a la primera etapa de planificación sanitaria y tiene por objeto evaluar la salud de una colectividad; así como proponer alternativas viables y factibles para la resolución de los mismos (3); es una técnica que permite analizar la situación de salud de una comunidad, determinar problemas y necesidades de salud,así como caracterizar sus factores determinantes, a través de un proceso que no concluye hasta que se encuentran soluciones a los problemas detectados. Existen diferentes tipos de diagnósticos de salud poblacional, los cuales se pueden basar en la perspectiva de los sistemas y servicios de salud o en un enfoque de participación comunitaria. Dentro del segundo enfoque se encuentra el Diagnóstico de Salud Participativo, el cual se sustenta en la Investigación Participativa Basada en la Comunidad, que se caracteriza por dar protagonismo a los actores sociales, integrándolos en el proceso de investigación, no sólo como personas que responden a encuestas, sino como destinatarios de los eventuales cambios en la sociedad. Los convierte en participantes activos durante la administración de cuestionarios, en la selección de entrevistados calificados con criterios de relevancia y también les permite participar en otras etapas del estudio y sugerir modificaciones para mejorar. Ésta colaboración comunitaria es más próxima a un trato entre iguales, entre los que llevan la responsabilidad del proyecto y la comunidad, integrando así el conocimiento y la acción para el mutuo beneficio de las partes (4).

El enfoque de estudio desde la perspectiva de la comunidad, considera a la misma como fuente de información y solución de su problemática, mientras que la perspectiva de los sistemas y servicios de salud se centra en las características demográficas, indicadores más relevantes y los recursos con que cuenta la población para buscar resolver sus problemas de salud presentes y futuros (5). La finalidad de los diagnósticos de salud, independientemente del enfoque, es poder emitir un juicio de valor en torno al proceso salud-enfermedad en relación a las necesidades, sentidas, expresadas y normativas $(6,7)$.
El Diagnostico de salud Participativo que a continuación se presenta, se elaboró en el sector IV de la comunidad de Dzityá, Yucatán, México y tiene como finalidad describir cada una de las fases que permitieron un acercamiento comunitario en busca de identificar los determinantes que afectan las condiciones de salud de la población desde la perspectiva de la comunidad. Inicialmente se detectó que en la población no existía un diagnóstico de Salud Participativo que contemplara los determinantes de la salud y los recursos y servicios con los que cuenta la comunidad para atender sus padecimientos.

El objetivo del presente estucio fue identificar los determinantes que afectan las condiciones de salud de una población desde la perspectiva de los actores sociales.

\section{MATERIAL Y METODOS}

En los meses de enero a abril de 2014 se realizó un diagnóstico de salud participativo en la localidad de Dzityá, perteneciente al municipio de Mérida, Yucatán, México. El estudio fue exploratorio y basado en la comunidad. Para la colecta de datos se incluyeron técnicas cuantitativas y cualitativas.

La información cuantitativa se obtuvo de 69 viviendas pertenecientes a uno de los sectores de la comunidad. Para la selección del sector se recabaron opiniones de líderes comunitarios y de salud de Dzityá; fueron ellos quienes, desde su perspectiva, propusieron realizar el diagnóstico en el sector IV, dadas las características sociales del lugar; mayor concentración de casas habitadas, y la cercanía con establecimientos identificados por la comunidad.

El muestreo de las viviendas fue aleatorio con arranque sistemático, se empleó la técnica de mapeo para localidades con manzanas definidas (8). Una vez seleccionada la vivienda, independientemente del número de personas que habitaban el hogar, por conveniencia se seleccionó a la persona informante para establecer un primer contacto, verificando que cumpliera los criterios de inclusión para la aplicación de una encuesta que exploró datos sociodemográficos, daños a la salud, características de la vivienda, prevención y detección de enfermedades por grupos de edad, manejo delagua, eliminación de excretas y saneamiento básico.

La información cualitativa se obtuvo del análisis del discurso (9) de 13 entrevistas semiestructuradas, y 2 ejercicios de cartografía social (10). Las entrevistas y la cartografía se realizaron en lugar, fecha y hora acordados 
entre los informantes y el equipo de trabajo, todas se audio grabaron y posteriormente se transcribieron textualmente en un procesador de textos. Para las entrevistas se diseñó una guía que exploró la percepción sobre los determinantes y daños a la salud, así como recursos con los que cuenta la comunidad para la atención sanitaria.El número de informantes se determinó hasta alcanzar la saturación teórica. La cartografía permitió identificar fortalezas, oportunidades, debilidades y amenazas que los escolares y jóvenes perciben en la comunidad.

La metodología para el Diagnóstico se dividió en 6 fases, durante cada una de ellas se realizaron visitas sucesivas a la comisaría.Las actividades se centraron en fomentar la participación de la población para la elaboración del diagnóstico, así como a la recopilación de información y análisis de la misma.

Las personas que participaron en el diagnóstico otorgaron su consentimiento de participación voluntaria; en el caso de los menores de edad, se contó con el asentimiento verbal y el consentimiento por escrito de sus tutores.

\section{RESULTADOS}

\section{Fase I. Preparación y acceso al campo.}

En esta fase se revisaron fuentes de información oficiales y locales para obtener un panorama general de la localidad. Primero se realizó un acercamiento informal con las autoridades de la comunidad y un recorrido por la zona de estudio (11). Estas actividades se apoyaron de una guía de observación, que abordó distintos aspectos de la Comunidad (Tabla 1), y la construcción de un mapa de la localidad. (12).

En esta etapa, el equipo de trabajo se presentó ante las siguientes autoridades; el comisario municipal, el comisario ejidal y el responsable del centro de salud. La finalidad de esta etapa fue establecer un primer contacto con la comunidad e identificar entornos potenciales para la recolecta de datos.

\section{Fase II. Recolecta de datos.}

En esta fase se obtuvieron datos primarios a partir de las 69 encuestas aplicadas en los hogares, 13 entrevistas semiestructuradas y 2 ejercicios de cartografía social. Los datos obtenidos se triangularon con la información obtenida en la fase I.
En las entrevistas semiestructuradas participaron; dos promotoras de salud, el director y la enfermera del centro de salud, la k'am palde Dzityá, el comisario municipal, el comisario, dos policías, el director de la escuela primaria, la directora de la escuela telesecundaria, y 2 artesanos que trabajan el tallado de piedra dura (Figura 1).

En la cartografía participaron 14 niños de la primaria y 12 jóvenes de la telesecundaria. Los niños de la primaria dibujaron lo que más les gusta y lo que menos les agrada de la localidad (fortalezas y debilidades); los jóvenes de la telesecundaria, además de las fortalezas y debilidades, identificaron las amenazas y oportunidades presentes en la comunidad. En ambos grupos intencionalmente se busco la participación de hombres y mujeres. Al inicio de cada sesión se explicó la importancia de que los niños y jóvenes participen en el diagnostico comunitario, se realizaron dinámicas de presentación y se proporcionaron los materiales necesarios para que los participantes plasmaran sus dibujos. Al final de la sesión los participantes presentaron sus trabajos ante sus compañeros.

\section{Fase III. Análisis de los datos.}

Para analizar los resultados cuantitativos se construyó una base de datos en el programa Excel 2010 y se utilizó estadística descriptiva para su interpretación. Los datos cualitativos fueron analizados a través de matrices manuales siguiendo los pasos sugeridos por Taylor y Bogdan, en su propuesta para el análisis del discurso (9). La cartografía se analizó a través de las imágenes y los testimonios que los participantes identificaron como espacios de riesgo y de fortaleza dentro de la comunidad. Los problemas y grupos identificados por la comunidad se presentan en la tabla 2.

\section{Fase IV. Priorización y validación social.}

En esta fase se cotejaron los resultados identificados desde el punto de vista epidemiológico con las necesidades expresadas por la comunidad. A través del método de Hanlon las mujeres de la comunidad indicaron que el problema prioritario a resolver eran las infecciones respiratorias causadas por las partículas de polvo de piedra originadas de la actividad artesanal.

Debido a que Dzityá es una comunidad en la que la mayoría de los hombres son artesanos que trabajan la madera y la piedra y sus talleres se encuentran en el centro del poblado, se observó durante las visitas a la comunidad, una gran cantidad de polvo blanco en calles, árboles, casas y patios. Las personas que regresan por las tardes del trabajo 
Tabla 1. Elementos de la guía de observación aplicada en la primera visita a la comunidad.

\begin{tabular}{ll}
\hline \multicolumn{1}{c}{ Elementos } & \multicolumn{1}{c}{ Conceptualización } \\
\hline Tiempo de traslado y rutas de acceso. & $\begin{array}{l}\text { Distancia y tiempo de la ruta Mérida-Dzi- } \\
\text { tyá. (Lugar donde se encuentra el grupo } \\
\text { de investigación hasta la zona de estudio). }\end{array}$ \\
Saneamiento básico. & Condiciones higiénicas del ambiente que \\
& se pueden apreciar por observación.
\end{tabular}

Saneamiento básico. se pueden apreciar por observación. ciones en las que se encuentran los domicilios, así como el conjunto de prestaciones y productos proporcionados por el Estado.

Características de la vivienda y servicios públicos.

Organizaciones sociales.

Servicios para la atención a la salud.

Espacios de recreación y deporte.

Dinámica poblacional.

Acceso y disponibilidad de alimentos.

Espacios y establecimientos para la compra-venta o intercambio de alimentos.

Instituciones o agrupaciones que trabajan para socializar y brindar servicios/atención a la gente que pertenece a ellos.

Establecimientos públicos, privados y actores sociales que ofrecen prestaciones sanitarias.

Sitios utilizados para la recreación, pasatiempo, placer, diversión o ejercicio físico.

Tamaño, composición y distribución espacial de las poblaciones y las variables que modifican sus dimensiones.
Materiales, tipo de construcción y condi-

Variables incluidas

- Vehículo personal: tiempo recorrido en

hr./min./seg., kilómetros recorridos, com-

bustible invertido, rutas de acceso.

- Transporte público: Tiempo recorrido en $\mathrm{hr} . / \mathrm{min} . / \mathrm{seg}$., rutas de acceso.

-Manejo de residuos sólidos: basura tirada en las calles, tiraderos oficiales/clandestinos y servicio de recolección.

-Fauna callejera: felinos/caninos y animales de traspatio.

-Abastecimiento de agua: presencia y características de pozos y cenotes de agua ${ }^{1}$.

-Material y tipo de la casa.

-Condiciones de la vía pública: pavimen-

tación, presencia de lotes baldíos, drenaje,

alumbrado y transporte público.

-Medios de información y comunicación: telefonía, televisión abierta y de paga, correo, periódicos/medios escritos, radio, telegrafía e internet.

Presencia de guarderías, escuelas, organizaciones de la sociedad civil, grupos de apoyo, congregaciones religiosas, cantinas, bares, trabajo sexual, restaurantes, hoteles, instituciones gubernamentales, grupos organizados para actividades culturales (fiestas populares), y actividades recreativas.

Presencia y funcionamiento de centros de salud, concurrencia, ambulancias, medios de traslado en caso de urgencia, medicina alternativa, medicina tradicional, consultorios, farmacias, y laboratorios.

Presencia, condiciones y características de espacios al aire libre, parques, canchas, y clubes deportivos.

-Grupos de edad.

-Género.

-Actividad económica (agrícola, comercio).

-Signos de migración: (marcas y tipos de coches y/o electrodomésticos, casas construidas con influencia norteamericana).

Presencia y características de tiendas, (incluyendo las de participación estatal mayoritaria), vendedores ambulantes, mercados, verdulerías, clubs de nutrición, huertos, árboles frutales y programas públicos de abasto, alimentación y nutrición.

Los cenotes son grandes depósitos naturales de agua de manantial, con características geológicas especiales, propio de la península de Yucatán (México) y otras partes de América. Los antiguos mayas utilizaban los cenotes como un lugar de culto. 
Tabla 2. Problemas y grupos identificados por la comunidad.

\begin{tabular}{|c|c|c|c|c|}
\hline $\begin{array}{c}\text { GRUPO } \\
\text { POBLACIONAL }\end{array}$ & $\begin{array}{c}\text { ENFERMEDADES } \\
\text { AGUDAS }\end{array}$ & $\begin{array}{c}\text { ENFERMEDADES } \\
\text { CRÓNICAS }\end{array}$ & $\begin{array}{l}\text { PROBLEMAS } \\
\text { SOCIALES }\end{array}$ & $\begin{array}{c}\text { PROBLEMAS DE } \\
\text { SANEAMIENTO } \\
\text { AMBIENTAL }\end{array}$ \\
\hline NIÑOS & $\begin{array}{c}\text { Diarreas } \\
\text { Infecciones } \\
\text { respiratorias }\end{array}$ & Obesidad & ---- & Partículas de polvo \\
\hline ADULTOS & $\begin{array}{l}\text { Infecciones } \\
\text { respiratorias }\end{array}$ & $\begin{array}{c}\text { Diabetes } \\
\text { Hipertensión } \\
\text { Hipercolesterolemia } \\
\text { Obesidad }\end{array}$ & ---- & $\begin{array}{l}\text { de piedra genera- } \\
\text { das de la actividad } \\
\text { artesanal. } \\
\text { Basura. }\end{array}$ \\
\hline JÓVENES & $\begin{array}{l}\text { Infecciones } \\
\text { respiratorias }\end{array}$ & ----- & $\begin{array}{l}\text { Alcoholismo } \\
\text { Embarazos a } \\
\text { temprana edad }\end{array}$ & Perros callejeros. \\
\hline
\end{tabular}

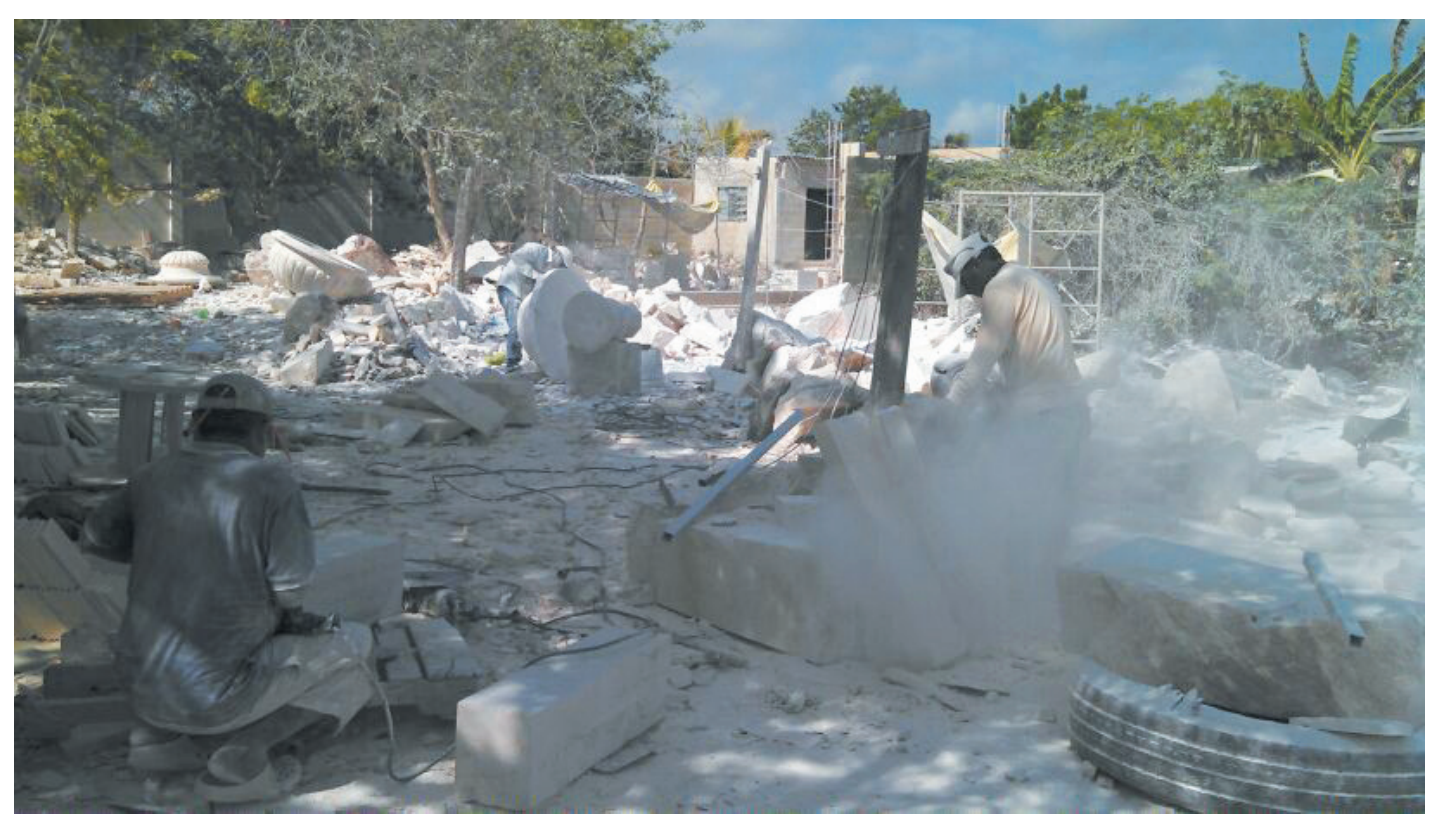

Figura 1. Hombres de la comunidad de Dzityá, Yucatán trabajando en la elaboración de artesanías a partir del labrado de piedra. Fuente: Elaboración propia, febrero 2014. (Footnotes) 1

lo hacen con mucho polvo en su cuerpo. A continuación se presenta un testimonio recogido de las entrevistas que mejor ejemplifica esta situación:

“.... los señores] pasan todos empolvados, parecen fantasmitas todos blancos (risa), es que trabajan la piedra y por tanto polvo que hay les da como un tipo de tos. Por ejemplo, uno de mis cuñados no sé si siempre va con el doctor para que le haga una limpieza en su nariz, incluso veo que utiliza mucho ese spray para la nariz... " (Mujer de 43 años, Dzityá, Yucatán).

\section{Fase V. Difusión de resultados.}

Esta fase tuvo como finalidad la devolución de los resultados a la comunidad. Se convocaron subgrupos de mujeres, profesores, personal de salud, autoridades municipales, niños y jóvenes, para discutir propuestas de solución al problema seleccionado. Se observó poca participación de los pobladores en esta etapa.

\section{Fase VI. Diseño de la intervención.}

En esta fase se buscó conjuntar los saberes del grupo de investigación con los saberes de la comunidad, partiendo del problema principal que fue la alta incidencia de enfermedades respiratorias. A través de la metodología del marco lógico, se diseñó una intervención educativa enmarcada dentro de la propuesta de promoción de la salud, para la prevención de enfermedades respiratorias en artesanos adultos de Dzityá, Yucatán. Este grupo fue 
seleccionado ya que era el más afectado por su prolongada exposición a las partículas de polvo de piedra.

\section{DISCUSIÓN}

En la fase I se realizó un acercamiento informal con las autoridades de la comunidady un recorrido por la zona de estudio con apoyo de una guía de observación. La observación directa y el contacto con las autoridades locales son actividades básicas que el personal de salud debe realizar para establecer alianzas con los pobladores y elaborar con mayor probabilidad de éxito, programas de atención dirigidos a la comunidad, tal como se demuestra en otro estudio de la misma naturaleza, donde se realizaron ambas actividades para establecer un primer contacto con la comunidad (13).

En la fase de recolecta de datos se emplearon técnicas con diferentes enfoques, ya que la literatura señala que la explicación de los problemas en la comunidad se basan en las estimaciones cuantitativas (indicadores) y cualitativas (valoración de la comunidad) que influirá en la determinación del riesgo actual y en la predicción del futuro (14). En este trabajo, ambos aspectos fueron considerados en la fase de recolecta de datos, al combinar técnicas cuantitativas y cualitativas, para asegurar el éxito de las intervenciones futuras.

Fueron varios los problemas detectados por la comunidad; sin embargo, en la fase de priorización, las mujeres seleccionaron como problema relevante, la contaminación ambiental generada por las partículas de polvo de piedra originadas de la actividad artesanal; lo anterior debido a que Dzityá es una comunidad principalmente de hombres artesanos que trabajan la madera y la piedra y sus talleres se encuentran en el centro de la comunidad, liberando grandes cantidades de polvo blanco, manifestado también en los testimonios de los habitantes de la comunidad. En la fase de priorización se empleo el método de Hanlon, ya que en otros trabajos ha sido ampliamente recomendado durante el proceso de toma de decisiones, porque permite a la comunidad establecer las prioridades ante los problemas que ha identificado, así como por su fácil aplicación y comprensión para la población (14).

Cuando se trató de la devolución de los resultados a la comunidad, se tuvo poco poder de convocatoria, a pesar de que se les invitó personalmente; según los informantes la inasistencia se debió a problemas con la organización de su tiempo. Es importante señalar que las mujeres fueron las que apoyaron en la mayoría de las etapas y principalmente durante la priorización de problemas, esto corresponde al interés que demuestran en las actividades que se organizan en la comunidad y por disponer de más tiempo que sus esposos para asistir a las reuniones. Esta situación es similar a lo reportado en otros trabajos donde la participación masculina ha sido poca o nula; a pesar que se han desarrollado estrategias como programar actividades en horarios y sitios accesibles para que los hombres puedan participar (15).

Finalmente,relacionarlos saberes del grupo de investigación con los saberes de la comunidad, se diseñó una intervención educativa enmarcada dentro de la propuesta de promoción de la salud, para la prevención de enfermedades respiratorias en artesanos adultos de Dzityá, Yucatán, debido a que era el grupo más vulnerable a este problema. En el ámbito de salud, estamos de acuerdo con otros autores cuando señalan que en los trabajos comunitarios se busca realizar un esfuerzo conjunto con la población y aplicar a la vez una metodología que pueda ser incorporada por la misma comunidad; en donde las estrategias deben ser claramente establecidas y detalladas a fin de facilitar la apropiación del recurso por parte de la comunidad, ya que es ella quien debe implementar programas de desarrollo y evaluar la pertinencia de las mismas (16).

\section{CONCLUSIONES}

La participación comunitaria es trascendental en la elaboración de diagnósticos de salud, ya que representa el insumo primario para la puesta en marcha de una efectiva planificación sanitaria. En ese sentido, si se pretende impulsar el desarrollo de las comunidades a través de su participación en la gestión de políticas públicas, se deberá reorientar el abordaje comunitario, permitiendo que la población se involucre de forma activa en la planificación, ejecución y evaluación de las etapas del proceso del diagnóstico.

En la comunidad de Dzityá, se encontraron diferentes comorbilidades y factores de riesgo de tipo ambiental y social que pueden ser modificables con intervenciones eficientes y oportunas con enfoque de promoción a la salud, mediante la voluntad política y la acción conjunta del sector salud, escolar y la participación comunitaria.

En este trabajo se aprecian avances en cuanto al acercamiento y reconocimiento de la participación comunitaria, sin embargo es conveniente señalar, que no hay una única forma de abordaje, ya que cada población es diferente en cuanto a cultura, organización, y nivel de participación. En ese sentido, es necesario empatizar con la comunidad 
y considerar aquellos factores que pueden afectar positiva o negativamente su desarrollo, para poder identificar sus necesidades, aproximarnos a su realidad y comprender sus actitudes.

Es importante dar continuidad a proyectos de investigación dirigidos a grupos específicos de la población de Dzityá. Explorar si existe contaminación ambiental a causa de las partículas de polvo de piedra y el ruido emitido en los talleres artesanales, expresar la magnitud y trascendencia del problema, si es que lo hubiera, y con base a ello justificar la generación de iniciativas, basadas en la comunidad, que contribuyan al mejoramiento de su salud.

\section{Agradecimientos}

Los autores expresan su sincero agradecimiento a los habitantes de la comunidad de Dzityá; niños, jóvenes, padres de familia, artesano, autoridades municipales, escolares y de salud. A los estudiantes de la especialización en salud pública de la Universidad Autónoma de Yucatán, por su disponibilidad y compromiso en la elaboración de este proyecto.

\section{REFERENCIAS BIBLIOGRAFICAS}

1. Sanmartí L. La salud y sus determinantes. Anthropos: Boletín de información y documentación. 1991; 118: 32-38.

2. Frenk J. La salud de la población: hacia una nueva salud pública. Fondo de Cultura Económica; 2000.

3. Pineault R, Daveluy C. La planificación sanitaria: Conceptos, métodos, estrategias. Segunda edición. Barcelona: Madisson, S.A; 1995.

4. Donabedian A. Los espacios de la salud: aspectos fundamentales de la organización de la atención médica. Fondo de Cultura Económica; 1988.

5. Márquez S, Arenas, L. Aspectos teóricos y conceptuales del diagnóstico de salud poblacional. En: Arenas L, Sosa D, Corrales T. Experiencias de acercamiento comunitario y participación de la población en salud. México: INDESOL, SEDESOL, INSP; 2008.

6. San Martín H. La crisis mundial de la salud. 2nd ed. Madrid: Editorial Ciencia 3;1985.

7. Pineault R, Daveluy C. Determinación de necesidades. En: Pineault R, Daveluy C. La planificación sanitaria. Conceptos métodos, estrategias. Barcelona: Masson; 1987. p. 43-203.

8. Instituto Nacional de Estadística, Geografía e Informática. Manual de la entrevistadora. Encuesta Nacional de la Dinámica Demográfica 1997 (ENADID 1997). México: INEGI; 1997.

9. Taylor S, Bogdan R. Introducción a los métodos cualitativos de investigación. 3ra ed. Barcelona: Paidós; 1996.

10. García C, Colombia E. La experiencia de cartografía social en la fase piloto del proyecto Barrios del Mundo. Estudio preliminar; 2003.

11. Rodríguez G, Gil G, García E. Acceso al campo: Vagabundeo. En: Metodología de la Investigación Cualitativa. Aljibe; 2000. p. 72-74.

12. Rodríguez G, Gil G, García E. Construcción de mapas. En: Metodología de la Investigación Cualitativa. Aljibe; 2000. p.113-117.

13. Cuba A, Arancibia F, Machado R, Flores M, Flores R, Flores S. Diagnóstico de salud comunitaria (Estudio situacional del barrio ENTEL UV. 77, Santa Cruz de la Sierra). Univ Cienc Soc. 2009; (Citado el 02 de Enero del 2015); 1(1):19-22. Disponible en: http://www.revistasbolivianas. org.bo/scielo.php?script $=$ sci_arttext\&pid $=\mathrm{S} 8888$ $88882009000100004 \& \operatorname{lng}=\mathrm{pt}$

14. Borroto-Chao R, Liz-Álvarez M. Diagnóstico comunitario de la situación de salud. Revista Cubana de Medicina General Integral. 1996; 12 (3): 289-294.

15. Alvares A, González M, López C, et al. Diagnóstico Integral de Salud Poblacional en Pedro Amaro, Jojutla, Morelos 2013. México: INSP; 2013.

16. Mori-Sánchez M. Una propuesta metodológica para la intervención comunitaria. Liberabit. 2008; 14 (14): 81-90.

\section{Correspondencia}

Didier Francisco Aké Canul.

Facultad de Enfermería, Universidad Autónoma de Yucatán, México. C. $90 \mathrm{~s} / \mathrm{n}$ x 59 y 59a Contiguo al Hosp. O’Horán Apdo. Postal No. 971. Mérida, Yucatán, México. correo electrónico: didier.ake@correo.uady.mx. Tel:. (9999) 24-22-16, Ext. 1013

Fecha de Recepción: 14 de febrero del 2015.

Fecha de aceptación: 10 de junio del 2015. 